\title{
INTERNACIONALIZACIÓN EN LAS BODEGAS FAMILIARES: UNA APLICACIÓN EMPÍRICA EN EL SECTOR DE LA RIOJA
}

\author{
Rubén Fernández Ortiz \\ Departamento de Economía y Empresa, Universidad de La Rioja, España \\ ruben.fernandez@unirioja.es \\ Guadalupe Fuentes Lombardo \\ Departamento de Administración de Empresas, Contabilidad y Sociología Universidad de Jaén, España \\ gfuentes@ujaen.es \\ María Leonor González Menorca \\ Departamento de Economía y Empresa, Universidad de La Rioja, España \\ leonor.gonzalez@unirioja.es
}

\section{RESUMEN}

El objetivo de este trabajo es analizar los motivos que llevan a las bodegas familiares de Rioja (España) a implantar un proceso de internacionalización. Del mismo modo, se estudian aquellos factores relacionados con la dotación de recursos intangibles de estas empresas que pueden ser la fuente de la ventaja competitiva de la compañía en el mercado de destino o, de otro lado, dificultar y limitar su estrategia internacional.

El estudio empírico se ha realizado sobre un total de veinte empresas pertenecientes a la Asociación de Bodegas Familiares de Rioja. En concreto, se ha realizado entrevistas en profundidad a directivos familiares y no familiares de estas bodegas, relacionadas con los objetivos de investigación aquí planteados.

Los resultados muestran que aquellas empresas con "un mayor carácter familiar" presentan un compromiso internacional menormedido a partir del porcentaje de ventas en el extranjero. Además, existen entre las compañías diferentes argumentos a la hora de justificar su internacionalización y la dotación de recursos intangibles para afrontarla también presenta determinadas diferencias.

PALABRAS CLAVE: INTERNACIONALIZACIÓN, EMPRESA FAMILIAR.

\section{ABSTRACT}

This work analyses the reasons why family businesses in La Rioja (Spain) implement internationalization processes. Furthermore, it studies the factors linked to the intangible asset pool, which may be the companies' source of competitive advantage in the target market or, on the contrary, hinder and restrain their international strategy.

The empirical study was performed on a sample of twenty companies that belong to the Association of La Rioja Family Wineries. In short, a series of in-depth interviews, based on this study's research objectives, were carried out on these companies' directors, whether they are family or not.

The results reveal that businesses with "a greater family nature" have a lower international commitment according to their percentage of international sales. Moreover, these businesses use different arguments to justify their internationalization and the intangible asset pool to implement it also shows certain differences.

KEYWORKS: INTERNATIONALIZATION, FAMILY BUSINESS

\section{INTRODUCCIÓN}

La estrategia de internacionalización de la empresa familiar presenta determinadas peculiaridades (Zhara, 2003; Fernández \& Nieto, 2005) como resultado de la conjunción de tres sistemas distintos (familia, propiedad y empresa) (Vallejo, 2003). 
Hasta la fecha, no han sido muchos los trabajos dedicados al estudio de la internacionalización de las empresas familiares (Gallo \& García-Pont, 1996; Okoroafo, 1999; Zahra, 2003; Fernández \& Nieto, 2005), a pesar de que en la última década han aumentado notablemente.

En general, estos estudios se centran en el análisis de los factores que favorecen y restringen el proceso de internacionalización en este tipo de empresas (Gallo \& Sveen, 1991); así como en el gran esfuerzo y cambio que supone el desarrollo e implantación de esta estrategia necesaria para la supervivencia de las empresas familiares (GaIlo \& Luostarinem, 1993; Nieto, 1999).

Paradójicamente, la carencia de estudios sobre la empresa familiar no se corresponde con la enorme importancia económica que estas empresas presentan, tanto en la generación de riqueza y empleo, como por ser uno de los principales agentes de la economía en el ámbito nacional e internacional (Leach \& Bogod, 1999; Romano et al., 2000). Las características de las empresas familiares, que las diferencian del resto (Kets de Vries, 1996; Ibrahim \& Ellis, 1994; Donckels \& Lambrecht, 1999), hacen que cualquier opción estratégica que deseen abordar pueda revestir para ellas unas particularidades únicas y específicas (Westhead, 1997), tal y como puede ser el caso de la estrategia de internacionalización.

El trabajo realizado por Fuentes (2006) sobre la influencia del carácter familiar de la empresa en su estrategia de internacionalización, aplicado al sector vitivinícola con denominación de origen en España, pone de manifiesto algunas diferencias en los motivos que llevan a estas empresas a desarrollar su expansión internacional en comparación con empresas no familiares. Este estudio tenía su base empírica, fundamentalmente, en las denominaciones de origen del ámbito de Jerez, y por tal motivo, una de las futuras líneas de investigación presentadas era extender a otras denominaciones de origen españolas los objetivos de investigación planteados en el trabajo con el propósito de analizar si se obtienen o no resultados similares. El trabajo que aquí presentamos se orienta en esta línea de investigación.

El objetivo de este estudio es analizar las motivaciones que llevan a la empresa familiar a implantar y desarrollar un proceso de internacionalización, dentro del sector vitivinícola con denominación de origen Rioja. En concreto, pretendemos conocer los argumentos que justifican la internacionalización de las empresas que forman parte de la Asociación de Bodegas Familiares de Rioja, así como las fortalezas, debilidades y factores que favorecen o limitan la actividad internacional de estas compañías y que se relacionan con su dotación de recursos intangibles.

Para alcanzar el objetivo propuesto en este trabajo hacemos uso de la metodología cualitativa y de la técnica de investigación de la entrevista en profundidad. Ambas se consideran las más adecuadas cuando el investigador pretende profundizar en el porqué y el cómo de un determinado fenómeno social (Taylor \& Bogdan, 1992); en nuestro caso, conocer las posibles motivaciones que llevan a una empresa familiar a desarrollar su expansión internacional, así como la influencia de las peculiaridades de estas compañías en el desarrollo de su proceso de internacionalización.

El trabajo ha sido estructurado en cinco apartados, incluido este primero de carácter introductorio. El segundo apartado está destinado a la revisión y análisis de la literatura que ha estudiado la estrategia de internacionalización en la empresa familiar en relación a los objetivos de investigación presentados. El tercero describe la metodología empleada y las fases de trabajo desarrolladas hasta alcanzar los resultados de investigación que se exponen en el cuarto apartado. Finalmente, el último apartado está destinado a comentar las principales conclusiones que se desprenden del estudio y a exponer las limitaciones del mismo. 


\section{Internacionalización de la Empresa Familiar}

A pesar de ser un reto complicado para las empresas familiares, la internacionalización resulta una tarea necesaria para que la mayor parte de ellas no descuiden sus capacidades de competir a largo plazo.

La internacionalización de la empresa familiar puede presentar argumentos distintos a los de otras compañías que no revistan este carácter. Del mismo modo, las peculiaridades de este tipo de empresas pueden influir decisivamente en el éxito alcanzado con esta estrategia. Dentro de este apartado realizamos una revisión de la literatura sobre ambos aspectos.

\section{Motivos que explican el proceso de internacionalización de la empresa familiar}

La internacionalización empresarial ha sido analizada en la literatura por numerosos autores (Guillén, 2001; Durán, 2005; Rialp \& Rialp, 2005).
Así, nos encontramos con distintos enfoques teóricos tales como la teoría de la ventaja en propiedad (Caves, 1971; Hufbauer, 1975; Hymer, 1976), la teoría del comportamiento oligopólico (Knickerbocker, 1973), la teoría de la internalización (Buckley \& Casson, 1976) y la teoría ecléctica (Dunning, 1973); que explican fundamentalmente la inversión directa en el extranjero. Otras teorías consideran la estrategia de internacionalización como un proceso; la teoría del ciclo de vida del producto (Vernon, 1966), el modelo de Uppsala (Johanson \& Vahlne, 1977) y la teoría de la inversión secuencial en el extranjero (Kogut, 1983; Kogut \& Kulatilaka, 1993).

Trabajos más recientes (Knight \& Cavusgil, 2004; Jones \& Coviello, 2005; Rialp et al., 2005) explican la expansión internacional de las empresas desde su constitución, las cuales han venido a denominarse "born-globals".

Estos enfoques teóricos justifican la internacionalización empresarial basándose en numerosos motivos que recogemos, a modo de síntesis, en la tabla 1.

\section{MOTIVACIONES PARA LA INTERNACIONALIZACIÓN EMPRESARIAL}

\section{TEORÍA DE LA EXISTENCIA DE VENTAJAS EN PROPIEDAD}

- Control para eliminar la competencia

- Diversificación empresarial

- Posesión de ventajas en propiedad en ciertas actividades:

- Ventajas de economía de escala

- Ventajas de diferenciación del producto

- Ventajas absolutas de costes.

- Aumentar los beneficios transfiriendo al extranjero la experiencia de fabricar productos diferenciados (inversión horizontal).

- Evitar la incertidumbre oligopólica y crear barreras de entrada a nuevos competidores (inversión vertical).

- Búsqueda de menores costes de los factores productivos

- Aumentar la demanda en países con mayor nivel de renta 


\section{TEORÍA DEL COMPORTAMIENTO OLIGOPÓLICO}

- Expansión de empresas oligopólicas, que obligan a las empresas rivales a responder con idéntica estrategia.

\section{TEORÍA DE LA INTERNALIZACIÓN}

- Sustituir el mercado por la propia organización interna, debido a los altos costes de transacción derivados de la venta de algunos activos intangibles.

- Existencia de imperfecciones en los mercados de capitales

- Complementariedad y experiencia de un producto

- Obtener beneficios derivados de las ventajas de internalización y localización.

\section{TEORÍA ECLÉCTICA}

- Posesión de tres categorías de ventajas:

- Ventajas específicas en propiedad.

- Ventajas de la localización

- Ventajas de internalización.

\section{TEORÍA SOBRE EL CICLO DE VIDA DEL PRODUCTO}

- Por la amenaza de perder cuota de mercado en la etapa de madurez del producto.

- Por reducir costes de producción a nivel global, dirigiéndose a países en vía de desarrollo.

- Por obtener economías de escala en producción.

- Poseer ventajas monopolísticas derivadas de la diferenciación del producto.

\section{MODELO DE UPPSALA}

- El nivel de experiencia que la empresa posee y los conocimientos acumulados.

- El interés de adquirir un mayor compromiso en los mercados internacionales.

\section{TEORÍA DE LA INVERSIÓN SECUENCIAL EN EL EXTRANJERO}

- La ventaja que supone para la empresa el poder disponer de capacidad (activos tangibles e intangibles) a lo largo de distintos mercados.

\section{BORN GLOBALS}

- Factores ambientales (tipo de sector, contexto geográfico, redes internacionales), recursos intangibles de la empresa (capital tecnológico, capital relacional, capital humano), y determinadas capacidades internacionales (rutinas orientadas internacionalmente, procesos de aprendizaje, etc.) fomentaran el nacimiento de empresas con vocación internacional.

FUENTE: Elaboración propia.

De otro lado, la teoría de recursos y capacidades ha sido empleada en el estudio del desarrollo e implantación de numerosas estrategias corporativas. De entre ellas, podemos destacar por su relevancia en la competitividad empresarial, la diversificación de los mercados o internacio- nalización de la empresa (Tallman, 1991; Collis, 1991; Andersen \& Kheam, 1998). Desde este enfoque, la estrategia internacional de la empresa se justifica, entre otros, por la infrautilización de determinados recursos, la posesión de recursos intangibles que generan ventajas competitivas, 
y la creación de valor por el uso y transmisión de determinados recursos.

Para el caso concreto de la empresa familiar, coincidimos con Alarcón y Saldaña (1996) cuando se refieren a la necesidad de internacionalizar las actividades de este tipo de empresas, como un instrumento capaz de asegurar su supervivencia y crecimiento, convirtiéndose éste último en el objetivo fundamental de la salida al exterior de la empresa familiar (Claver et al., 2000, 2007). Además de éstos, son numerosos los motivos que la empresa familiar persigue mediante su proceso de internacionalización. Entre otros, se destacan:

- la necesidad de expandirse más allá de sus mercados domésticos (Yeung, 2000).

- el obtener un mayor conocimiento que les lleve a ser fuertes competidores en sus mercados locales cuando se percibe una disminución de las oportunidades de crecimiento en el mercado doméstico.

- el deseo de diversificar riesgos.

Estos aspectos pueden ser causas de la expansión internacional de estas empresas, al igual que lo son para el resto de empresas, pero debemos considerar que la mayor aversión al riesgo que parecen mostrar las empresas familiares (McConaughy et al., 2001) podría generar un deseo por reducirlo, y tal y como señalan algunos autores (Davis \& Harveston, 2000), se puede lograr la diversificación del riesgo empresarial actuando en distintos mercados geográficos.

De otro lado, la literatura revisada sobre empresa familiar nos muestra que una de las fortalezas de estas compañías radica en el hecho de presentar un fuerte grado de compromiso. En la medida en que exista el deseo de proyectar este compromiso fuera del mercado doméstico, podría justificarse la estrategia de internacionalización de la empresa familiar.
Además, el deseo de proporcionar trabajo dentro de la empresa a miembros de la familia que se desean incorporar al negocio, y que en ocasiones, no encuentran sitio en la estructura existente, así como, proporcionar oportunidades de promoción interna a directivos familiares y no familiares que deseen emprender nuevas actividades, serían otras motivaciones para desarrollar una estrategia de salida exterior en este tipo de empresas (Yeung, 2000).

\section{Influencia de las peculiaridades de la empresa familiar en su estrategia internacional}

Desde el enfoque de recursos y capacidades, la estrategia de internacionalización de la empresa familiar podría explicarse a partir de la posesión de ventajas en propiedad, destacando la importancia de la disponibilidad de recursos intangibles por parte de la empresa como la principal fuente de ellas. Los recursos intangibles pueden ser de varios tipos: humanos, tecnológicos, organizativos y relacionales. Pues bien, en cada uno de ellos, la empresa familiar posee unas características peculiares derivadas de su carácter que pueden potenciar o limitar su expansión internacional.

La teoría nos muestra cómo algunas de las principales fortalezas de la empresa familiar radican en el hecho de poseer conocimiento y experiencia del negocio (Donckels \& Lambrecht, 1999), compromiso (Horton, 1986), lealtad (James, 1999), confianza (Steier, 2001) y comunicación entre sus miembros (Ibrahim et al., 2001), incluidos los trabajadores, y la búsqueda de la satisfacción de éstos (Sorenson, 2000). Abundando en esta idea, Vallejo (2003) encuentra que los empleados de las empresas familiares muestran mayores niveles de lealtad, implicación e identificación, que se traducen en un mayor grado de compromiso con la empresa. Igualmente encuentra un mayor grado de cohesión entre los 
miembros de este tipo de organizaciones en relación a las no familiares. Así, el stock de recursos humanos con el que cuenta la empresa familiar presenta una serie de fortalezas, respecto al de las empresas que no revisten dicho carácter, que puede constituir la fuente de la ventaja competitiva en su proceso de internacionalización, siempre y cuando supere algunas de las debilidades que lo caracterizan tales como la existencia de nepotismo (que provoque la falta de una gestión profesionalizada y la dificultad de atraer a directivos externos) y/o discordias familiares que influyen en el negocio, y que conllevan a una falta de armonía entre los miembros de la organización, pues estas debilidades pueden obstaculizar cualquier proyecto estratégico que la empresa se proponga, así como su internacionalización.

Considerando los recursos tecnológicos de la empresa familiar, no han sido muchos los trabajos que han investigado la relación existente entre estas empresas y su tecnología. Así, encontramos un trabajo de Ward (1997) que nos aporta que en este tipo de empresas se presta una mayor atención al I+D, respecto de aquellas empresas que no son familiares. El conocimiento de la tecnología utilizada es una fortaleza de la empresa familiar, cuyos miembros conocen perfectamente los secretos industriales de los procesos productivos, con los que se han familiarizado a través de un proceso de aprendizaje de mucho tiempo. Gudmundson et al. (2003) destacan la mayor capacidad innovadora de la empresa familiar, en el sentido de que es capaz de iniciar y desarrollar nuevas ideas, como resultado de la mayor autonomía y creatividad de la que suelen gozar sus trabajadores. Chandler (1990), sin embargo, considera inadecuada e insuficiente la inversión en I+D y en nuevas tecnologías que realizan las empresas familiares, alegando las preferencias de la familia propietaria por cuidar y mantener financiado su privilegiado estilo de vida.

Atendiendo a los recursos organizativos con los que cuenta una empresa familiar, éstos también presentan una serie de peculiaridades derivadas del carácter de estas empresas. Por un lado, este tipo de empresas se caracteriza por poseer organizaciones confusas, con estructuras poco definidas y con falta de una clara división de tareas (Kets de Vries, 1993). Sin embargo, también debemos tener en cuenta que sus estructuras organizativas hacen gala de su flexibilidad, es decir, son menos jerárquicas y burocráticas que las no familiares, lo que a la vez permite responder de manera rápida y efectiva a los frecuentes cambios acaecidos en el entorno (Tagiuri \& Davis, 1996; Poza et al., 1997). Además, existe una escasa formalización del proceso de toma de decisiones (Daily \& Dollinger, 1992), lo que dota al negocio de una gran habilidad comunicativa y de eficiencia en la gestión, y en consecuencia, es probable que estas peculiaridades puedan flexibilizar el proceso de internacionalización de la empresa familiar si ésta logra superar algunos de los inconvenientes que la caracterizan tales como un relevo generacional no planificado, la falta de una estructura organizativa clara y un estilo de dirección autocrático.

Finalmente, los recursos relacionales de la empresa familiar, tales como reputación, imagen de la empresa, y sus relaciones con terceros pueden constituir la fuente de la ventaja competitiva en su expansión internacional. En este sentido, Hall (1992) muestra como la reputación, que representa el conocimiento y las emociones llevadas a cabo por individuos, sobre, por ejemplo, una gama de producto, puede ser muy importante en la obtención de ventajas competitivas vía diferenciación. En las empresas familiares, se presta una mayor atención al desarrollo de la marca (Ward, 1997), en muchas ocasiones esta marca o el nombre de la empresa está vinculado con el apellido familiar y es sinónimo incuestionable de calidad, es más, lo puede venir siendo durante generaciones. De este modo, podría explicarse la alta preocupación que estas empresas muestran por mantener y por cuidar la calidad y por prestar un excelente servicio para conseguir la satisfacción de sus clientes (Poza, 1995), así como, por su fuerte implicación con el entorno 
social en él que actúan, dando una gran importancia a las relaciones con los clientes, proveedores, distribuidores y mostrando una alta responsabilidad social (McWhinney, 1988).

A modo de resumen, mientras que los recursos tecnológicos de la empresa familiar no parecen ser la fuente de ventajas competitivas, los recursos humanos, organizativos y relacionales de es- tas empresas sí podrían ser el origen de las mismas. Por ello, en la figura 1 recogemos aquellos recursos intangibles (humanos, organizativos y relaciones) específicos de la empresa familiar que pueden favorecer la implantación y el desarrollo de su estrategia de internacionalización una vez superadas las debilidades que caracterizan a estas compañías, relacionadas también con su carácter familiar.

FIGURA 1

\section{RECURSOS INTANGIBLES DE LA EMPRESA FAMILIAR}

\section{RECURSOS INTANGIBLES}

RECURSOS TECNOLÓGICOS

RECURSOS HUMANOS

RECURSOS ORGANIZATIVOS

RECURSOS RELACIONALES
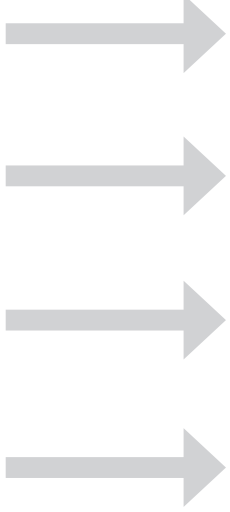

EMPRESA FAMILIAR

CAPACIDAD DE INNOVACIÓN, MENOR DOTACIÓN TECNOLÓGICA

CONOCIMIENTO, COMPROMISO,

IDENTIFICACIÓN, LEALTAD, ETC

FLEXIBILIDAD, COMUNICACIÓN, ESCASA FORMALIZACIÓN, ETC

IMAGEN, BUENAS RELACIONES CON LA COMUNIDAD, VISIÓN A L/P, ETC

FUENTE: Elaboración propia.

\section{METODOLOGÍA}

Tal y como exponíamos en la parte introductoria de este trabajo, la metodología utilizada en nuestro estudio empírico ha sido la cualitativa por ser considerada como la más adecuada cuando el investigador pretende profundizar en el por qué de un determinado fenómeno social. Además, usamos como técnica de investigación cualitativa, la entrevista en profundidad por el gran volumen de información que nos aporta sobre los objetivos de investigación recogidos en este estudio. En los apartados siguientes vamos a referirnos a cada una de las fases de trabajo desarrolladas en la parte empírica de la investigación.

\section{Selección de la muestra}

Dentro de la fase de diseño del trabajo resulta de vital importancia la selección de la muestra de empresas que participan en el estudio. En relación a este aspecto, se han elegido las empresas pertenecientes a la Asociación de Bodegas Familiares de Rioja. El principal motivo de esta elección es que este grupo de empresas se encuentra dentro de una de las más importantes denominaciones de origen españolas a nivel internacional, son de carácter familiar y en la actualidad están implantado o desarrollando una estrategia de internacionalización. Seleccionan- 
do empresas del sector que pertenezcan a alguna denominación de origen española podemos garantizar cierta homogeneidad en la calidad de los productos, para que no se convierta esta variable en el principal argumento que explique la expansión internacional de estas compañías. Las variables que se han considerado para analizar el carácter familiar de las compañías han sido la distribución de la propiedad del capital social de la empresa, la distribución de los puestos directivos y miembros del consejo de administración y el deseo de transmitir la empresa a la siguiente generación familiar. Finalmente, el interés que muestran estas compañías por su expansión internacional se pone de manifiesto por un reciente proyecto de investigación firmado entre la Asociación a la que pertenecen, la Universidad de La Rioja y la Universidad de Jaén, en el que se estudian los factores de internacionalización de las bodegas familiares de la Denominación de Origen Calificada Rioja.

En el estudio empírico participan un total de 20 empresas, entre las cuales se han realizado entrevistas a directivos familiares y no familiares durante el periodo de tiempo comprendido entre enero y abril de 2008.

\section{Recolección de la información}

La información utilizada para realizar el estudio empírico ha provenido de dos fuentes. En primer lugar se elaboró una ficha de empresa con información secundaria que provenía de distintas bases de datos empresariales, publicaciones periódicas del sector, páginas web de las empresas, ect. En segundo lugar, se realizaron entrevistas en profundidad a los directivos de las compañías.

A modo de síntesis, tanto la información recogida en la ficha de empresa, como las cuestiones planteadas en las entrevistas se relacionaban primero, con la caracterización de cada una de las empresas que participaron en el estudio (familiar/no familiar) y, segundo, con los motivos que las llevaban a desarrollar una estrategia de internacionalización.

Entre las primeras destacamos el porcentaje del capital de la empresa que poseían miembros de una misma familia, el porcentaje de puestos directivos y cargos del consejo de administración ocupados por miembros de la familia propietaria y las generaciones familiares que trabajaban en la empresa durante el periodo de tiempo en el que se realizó el estudio empírico.

Entre las segundas, se trató de limitar la importancia que los mercados exteriores tenían para la empresa; precisar cuáles, de las decisiones estratégicas que se tomaban, estaban orientadas al mercado exterior; evaluar las relaciones que la empresa mantenía con mercados internacionales en función de su estabilidad y frecuencia; obtener el porcentaje sobre el total de las ventas de la empresa en mercados distintos del local, comarcal, regional y nacional, en el caso de que su actividad internacional se limitase a la exportación, y/o la existencia de inversiones directas realizadas en mercados extranjeros; $y$, finalmente, determinar las motivaciones para desarrollar su proceso de internacionalización, aspectos favorecedores y aspectos limitadores de esta estrategia.

\section{RESULTADOS}

\section{Análisis y resultados de los datos recogidos en la ficha de empresa}

A partir de esta información se obtuvieron distintos porcentajes de ventas en el exterior de las empresas, que parecen relacionarse con aquellas variables a las que nos referimos anteriormente para considerar el carácter familiar o no de las compañías (propiedad, dirección y relevo generacional). Así, las empresas que presentan los menores porcentajes de ventas en el extranjero son aquellas en las que su carácter familiar es más acusado por encontrarse en segunda generación familiar, por tener el capital al cien por 
cien en manos de miembros de una misma familia y muy concentrado en pocas persona. Además, en estas compañías, tanto los puestos directivos como los cargos del consejo de administración son ocupados por miembros de la familia en su totalidad.

Sin embargo, aquellas empresas que presentan los mayores porcentajes de ventas en el exterior, superiores al veinte por ciento, se caracterizan por ser compañías de reciente creación o estar en tercera generación familiar, lo que implica haber superado los problemas del relevo generacional. Además, el capital se distribuye entre un mayor grupo de personas, en comparación con las anteriores empresas, e incluso observamos que se encuentra repartido entre personas que no pertenecen a la misma familia. Finalmente, los puestos directivos y los cargos del consejo de administración son ocupados no exclusivamente por personas pertenecientes a la misma familia.

De este modo, encontramos empresas con un mayor y un menor compromiso internacional, medido a partir del porcentaje de ventas en el mercado exterior, y que parece relacionarse con el grado de familiaridad de la compañía a partir de las variables a las que nos hemos referido anteriormente (ver figura 2).

\section{FIGURA 2}

\section{DESCRIPCIÓN DE LAS EMPRESAS: CARÁCTER FAMILIAR E INTERNACIONALIZACIÓN}
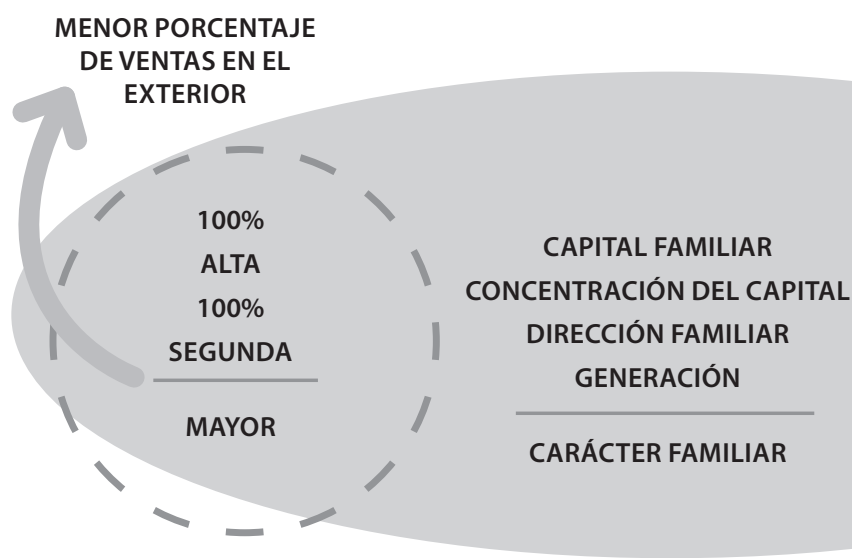

FUENTE: Elaboración propia

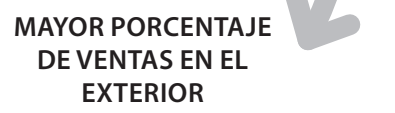

Así, aquellas empresas con "un menor carácter familiar" y mayor porcentaje de ventas en el exterior se caracteriza por tener la propiedad repartida entre al menos tres personas, en muchos casos, además, esta propiedad está compartida con personas que no pertenecen a la familia, son empresas que tienen la minoría de los puestos directivos ocupados por miembros de la familia y los cargos del consejo de administración compartidos por personas familiares y no familiares. Finalmente, son empresas que se encuentran en primera generación y que aún no se han enfrentado al relevo generacional o que ya han superado éste.

Sin embargo, las empresas con "un mayor carácter familiar", y que a su vez presentan los porcentajes de ventas en el exterior más bajos, tienen su propiedad al cien por cien en manos de miembros de una misma familia, en casi todos los casos. Dicha propiedad se encuentra muy concentrada o concentrada en pocas personas, 
los puestos de dirección están ocupados en su totalidad o mayoritariamente por familiares, al igual que ocurre con los cargos del consejo de administración. Finalmente, podemos observar, que muchas de las empresas que se encuentran en este grupo están en la actualidad en su segunda generación, por lo que pueden presentar problemas de sucesión más acusados.

\section{Análisis y resultados de los discursos recogidos en las entrevistas}

Una vez que hemos descrito las empresas que forman parte de nuestro estudio a partir de la información recogida en la ficha de empresa, en este apartado analizamos la información de las entrevistas y los resultados obtenidos. Así, nuestro principal material objeto de análisis es el discurso contenido en cada una de las entrevistas realizadas. Además, debemos señalar que el análisis de cada uno de los discursos individuales nos lleva también a encontrar las relaciones entre el total de los discursos formulados por todas las personas entrevistadas.

La preparación de nuestro análisis comenzó por una trascripción literal de las entrevistas y una lectura (escucha) varias veces repetida de todas las entrevistas realizadas. Tras esta fase, se inició una reconstrucción personal de la información contenida en las mismas, en la que establecimos las relaciones entre las distintas categorías que son objeto de nuestro interés.

En los subepígrafes siguientes, vamos a recoger los resultados obtenidos en cada uno de los objetivos de investigación planteados, a partir del análisis de los discursos de las entrevistas.

\section{Motivaciones para la expansión internacional de la empresa}

En el repaso de la literatura observábamos que eran numerosos los enfoques teóricos que explican el por qué de la internacionalización empre- sarial. En este apartado trataremos de identificar cuáles son los motivos por los que las empresas, que han formado parte de nuestro estudio empírico, quieren abrir su actividad más allá del mercado doméstico.

La saturación del mercado nacional es el principal motivo que justifica la expansión internacional esgrimido por las empresas del estudio. Esta saturación viene causada por el descenso del consumo per cápita (nuevas leyes, cambio de hábitos, etc.) y por el aumento de la competencia dentro de las fronteras nacionales. De ahí, que muchas empresas del estudio, se hayan decidido, desde su constitución, a realizar su actividad más allá del ámbito nacional, lo que unido a la actitud emprendedora de sus propietarios, las convierte en empresas born globals.

Otro motivo que justifica la expansión internacional es el hecho de que en el mercado internacional se obtiene un mayor margen de beneficio sobre un mismo producto. Muchas de las personas entrevistadas aseguran que dentro de nuestras fronteras, en muchas ocasiones, se fija el precio antes ni siquiera de conocer la calidad del producto. Sin embargo, el mercado internacional valora más los productos de calidad.

Además, estas empresas deciden exportar para poder aumentar su volumen de ventas. Este motivo está estrechamente relacionado con el primero, pues al comprobar que es muy difícil el crecimiento en el territorio nacional, optan por crecer en el extranjero. En relación al crecimiento de la empresa a través del desarrollo de la estrategia de internacionalización podemos afirmar que pese a ser un objetivo importante para todas las compañías entrevistadas, solo aquellas que presentan un menor carácter familiar realizan una planificación estrategica adecuada para alcanzarlo.

Existen otros tres motivos que justifican la expansión internacional: diversificación del mercado, deseo de adquirir una mayor reputación y prestigio, y mayor seguridad y seriedad en sus negocios. 
Con el primero de ellos, más presente en aquellas compañías que presentan "un menor carácter familiar", las empresas consiguen disminuir el riesgo. Así, este motivo confirma uno de los factores de tipo estratégico enunciado por Fernández-Ortiz (2005) y Fuentes (2006), como flexibilizadores de la internacionalización de las empresas familiares.

La reputación y el prestigio de vender en el extranjero sigue siendo un motivo importante, pues algunas empresas de la muestra consideran que mejoran su imagen cuando consiguen desarrollar su actividad más allá de su mercado doméstico, argumento éste más señalado en las compañías que se caracterizan por un mayor carácter familiar y entre los directivos familiares.

Por último, hay compañías que señalan la existencia de una mayor seguridad y seriedad en los negocios que realizan en el extranjero, comparándolos con aquellos otros realizados en el territorio nacional, lo cuál podría justificarse a partir de la enorme competencia que parece existir en el mercado nacional. Del mismo modo, los directivos de estas empresas argumentan que nunca han tenido problema de cobro con los países a los que exportan, algo que no pueden afirmar en el sector nacional.
En general, los resultados alcanzados en este objetivo de investigación son variados, tal y como eran los argumentos a los que aludían las teorías recogidas dentro de nuestro marco teórico al respecto. No obstante, los principales motivos del deseo de lograr esa expansión vienen provocados por la particular situación a la que se enfrenta el sector, con un clima competitivo muy asfixiante en el mercado más cercano.

\section{Valoración de los recursos intangibles de las empresas en su expansión internacional}

En este apartado, exponemos las principales fortalezas y debilidades de las empresas entrevistadas de cara a su expansión internacional, así como los principales factores que favorecen o limitan su estrategia de internacionalización y que se relacionan con la dotación de recursos intangibles que posee la empresa. De este modo, los recursos tecnológicos, humanos, organizativos y relaciones de las compañías que forman parte de este estudio pueden ser la fuente de la ventaja competitiva de la empresa en el mercado exterior o, de otro lado, limitar la expansión internacional de la empresa.

En la figura 3 sintetizamos la dotación de recursos intangibles que se ha observado en las empresas de la muestra.

FIGURA 3

\section{VALORACIÓN DE LOS RECURSOS INTANGIBLES}

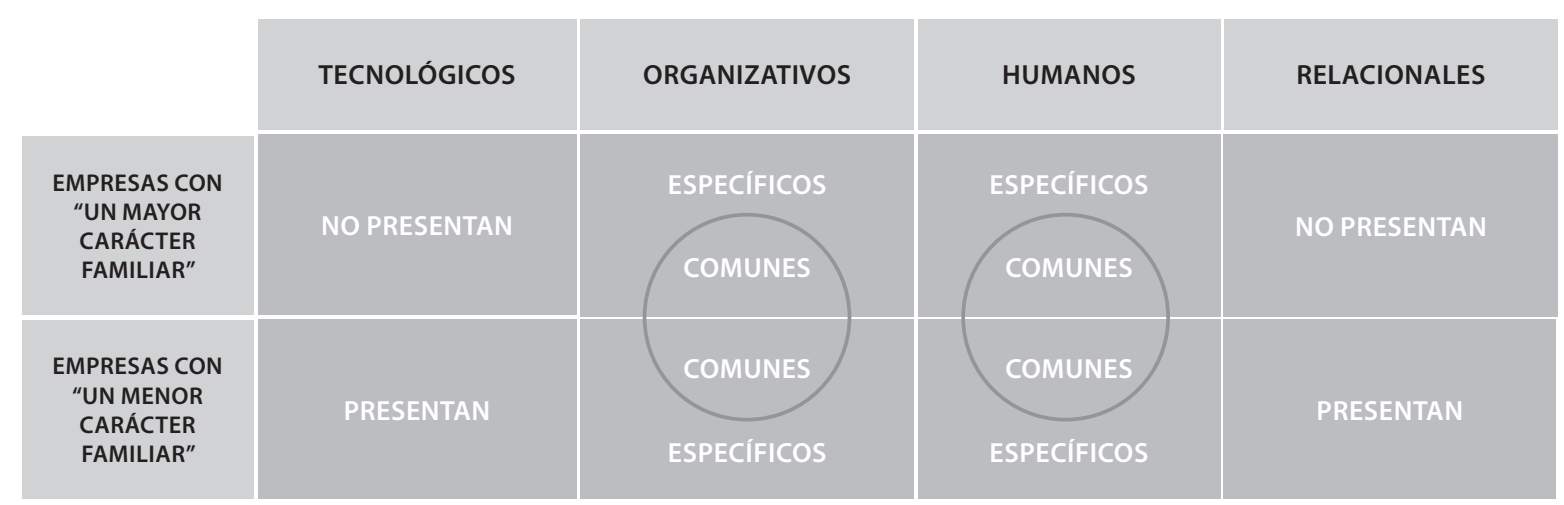

FUENTE: Elaboración propia. 
Analizando la posible influencia de esta dotación de recursos intangibles en la estrategia de internacionalización de las empresas, existen diferencias destacables.

En relación a la dotación de recursos tecnológicos en las empresas con "un mayor carácter familiar" ésta es prácticamente inexistente, mientras que entre los recursos tecnológicos que muestran las empresas con "un menor carácter familiar" nos encontramos con dotaciones tecnológicas, proyectos y gastos en I+D+i, patentes y dominios de internet.

Considerando los recursos organizativos de los dos tipos de empresas, las más familiares presentan como principal debilidad la falta de planificación del proceso de relevo generacional, así como una toma de decisiones muy concentrada en pocos miembros familiares con escasa cultura internacional. Además, existe una falta de especialización y sus estructuras organizativas son informales. Sin embargo, una de las principales fortalezas atribuidas a estas compañías se relaciona con su marcado carácter familiar pues poseen una gran flexibilidad y rapidez en la toma de decisiones. Como ya se ha mencionado antes, la mayoría de empresas son de dirección familiar, por lo que las decisiones no deben ser consultadas al consejo de administración, ya que está formado por los propios directivos, lo cuál les otorga esta ventaja. La flexibilidad se ve reflejada en diversos aspectos, como puede ser la adaptación del producto al cliente internacional, mecanismos de distribución, etc. Mientras que la toma de decisiones en las empresas menos familiares está más diluida, incluso compartida con personas ajenas a la familia, existe una mayor especialización y gozan de una estructura organizativa más formalizada. Finalmente, en estas empresas la toma de decisiones se comparte con personas externas a la familia con una amplia cultura, formación y experiencia internacional.
Atendiendo a la dotación de recursos humanos de estas compañías, se observa como la experiencia y la tradición familiar en el negocio constituye una auténtica fortaleza para todas las empresas del estudio. Si una familia lleva bastantes años, o incluso varias generaciones, trabajando en el mismo negocio, es señal de que conocen su funcionamiento, lo que le otorga unas garantías de seguridad de cara al cliente. En el sector del vino, este factor multiplica su importancia debido a que el know how resulta vital para obtener un producto de calidad. Sin embargo, también encontramos diferencias en la dotación de recursos humanos de estas empresas considerando el mayor o menor carácter familiar de la compañía. Así, los recursos humanos de las primeras se caracterizan porque sus propietarios exigen la adaptación de todos los miembros de la empresa a la cultura familiar, existe falta de especialización, poseen una escasa formación y experiencia internacional y el estilo de liderazgo podría describirse como autocrático y paternalista. De otro lado, estas empresas consideran que su principal fortaleza radica en la dedicación y el orgullo que la familia siente por la empresa. El hecho de que sea la propia familia la que dirija la empresa, es motivo de orgullo para sus propietarios. También perciben que es algo apreciado por los clientes, los cuales agradecen el trato directo con las personas que dirigen la empresa que visitan. Mientras que las empresas con "un menor carácter familiar", pese a que también exigen, en algunos casos, una adaptación de los directivos externos a la cultura familiar, el estilo de liderazgo es más participativo que en el caso anterior y sus miembros presentan actitudes empresariales emprendedoras y una alta formación y experiencia internacional. La motivación de los trabajadores de estas compañías se realiza a través de la fijación y consecución de objetivos y se valora mucho la creatividad de todos los miembros. Los directivos no familiares de estas empresas añaden a lo anterior el espíritu emprendedor y arriesgado de todo el equipo directivo (familiares o externos) y la satisfacción que supone para los empleados trabajar en estas empresas. 
La dotación de recursos relacionales que presentan las empresas ha sido diferente para las empresas con "un mayor carácter familiar" en comparación con aquellas otras que poseen "un menor carácter familiar". De este modo, las primeras realizan pocas inversiones en marketing, gozan de reconocimiento, imagen y reputación solo en su ámbito local o provincial, aunque consideran la importancia de mejorar estos aspectos a corto plazo en el ámbito nacional, y las relaciones que mantienen con instituciones públicas o privadas fuera de su localidad son prácticamente inexistentes. De otro lado, las segundas realizan elevadas inversiones en marketing considerando que dentro de este área es de vital importancia el papel de la familia, su imagen (ya que el reconocimiento a nivel internacional está muy relacionado con el apellido familiar), se plantean obtener certificados de gestión de la calidad y medioambientales, y mantienen relaciones directas y estrechas con distintas instituciones nacionales e internacionales. Del mismo modo, estas compañías están desarrollando nuevas actividades relacionadas con el enoturismo para diversificar sus actividades y llegar mejor al cliente.

\section{CONCLUSIONES}

Las peculiaridades que caracterizan a las empresas familiares, consecuencia del solapamiento que se produce entre dos sistemas distintos, familia y empresa (Lansberg, 1983), afectan a su dirección estratégica en general y a la estrategia de internacionalización en particular (Fernández \& Nieto, 2005).

El trabajo presentado se centra, precisamente, en el estudio de los motivos que llevan a la empresa familiar a implantar y desarrollar un proceso de internacionalización y al análisis de la influencia de las peculiaridades sobre esta estrategia.

El soporte teórico para realizar la investigación integra los argumentos que proceden de las teorías que analizan la internacionalización empresarial desde el enfoque económico y el enfoque de recursos y capacidades aplicado a esta estra- tegia y la revisión de la literatura que estudia las peculiaridades de la empresa familiar.

La revisión de las distintas aportaciones teóricas sobre la estrategia de internacionalización empresarial recogidas en el enfoque económico (Guillén, 2001) nos permite conocer cuáles son las principales motivaciones que llevan a la empresa a desarrollar una estrategia de internacionalización. Mientras que el estudio del enfoque de recursos y capacidades nos permite conocer aquellos recursos de carácter intangible (tecnológicos, humanos, organizativos y relacionales) que pueden considerarse, en muchas ocasiones, un activo estratégico para la empresa. Revisando la literatura sobre empresa familiar se han reconocido aquellos recursos de carácter intangible que pueden ser la fuente de la ventaja competitiva de la compañía en el exterior una vez superen las dificultades con las que estas empresas parecen contar y que se derivan de su peculiar carácter.

Para el análisis empírico de los objetivos de investigación se utilizó la metodología cualitativa por ser la más adecuada cuando se pretende profundizar en la comprensión, el por qué de un determinado fenómeno social (en nuestro caso, un proceso de internacionalización empresarial). Además, se usó, como técnica de investigación cualitativa, de la entrevista en profundidad pues sus rasgos diferenciales aseguran disponer de la información suficiente y adecuada para poder formular inferencias, llegar a conclusiones y describir con objetividad situaciones o fenómenos relacionados con el objetivo de esta investigación.

Las empresas que han formado parte de este estudio pertenecen al sector vitivinícola $y$, en concreto, son bodegas que forman parte de la Denominación de Origen Calificada Rioja y de la Asociación de Bodegas Familiares de Rioja.

Toda la información utilizada para obtener los resultados de la investigación ha provenido de las fichas de empresa y de los discursos contenidos en las entrevistas realizadas. 
A partir de los datos recogidos en las fichas de empresas se obtuvo, entre las empresas que participan en el estudio, dos grupos: aquellas cuyo porcentaje de ventas en el extranjero sobre el total es inferior al veinte por ciento y aquellas otras que superan este porcentaje. Además, del análisis se deriva una primera conclusión: el comportamiento internacional de las empresas, parece estar relacionado con "un mayor o menor carácter familiar" de las compañías.

Los motivos argumentados en la revisión del marco teórico para explicar la estrategia de internacionalización empresarial, coinciden, a excepción de algunas señaladas por las compañías con "un mayor carácter familiar", con aquellas obtenidas en los resultados: crecer, diversificar el riesgo, aumentar la cuota de mercado y obtener ventajas en márgenes.

Considerando la influencia de los recursos intangibles (tecnológicos, organizativos, humanos y relacionales) como fuente de ventaja competitiva en la implantación y desarrollo de la estrategia de internacionalización, los resultados obtenidos han sido diferentes en función del mayor o menor carácter familiar de la empresa.

Al tratarse de un estudio de carácter cualitativo, 20 muestras es un número apropiado y suficiente; sin embargo, una muestra mayor hubiera beneficiado la investigación en aspectos como la representatividad.

Debido a que el estudio se realizó dentro del marco de una asociación de bodegas familiares, no se puede establecer una comparativa con el comportamiento de las empresas no familiares del sector, lo que nos habría permitido conocer cómo afecta el carácter familiar o no de la empresa en los distintos aspectos abordados en este proyecto.

Finalmente, nos gustaría mostrar nuestro profundo agradecimiento a la Asociación de Bodegas Familiares de Rioja por habernos permitido llevar a cabo la labor investigadora aquí recogida.

\section{REFERENCIAS}

Alarcón, J.E. \& Saldaña, P. (1996). La carcoma del árbol generacional: Podar o abonar. Comunicación Presentada al VICongreso Nacional de ACEDE, pp. 867-881.

Andersen, O. \& Kheam, L.S. (1998). Resource-based theory and international growth strategies: An exploratory study, International Business Review, 7, (2), pp.163-184.

Buckley, J. \& Casson, M.C. (1976). The future of the multinational enterprise. London: McMillan.

Caves, R. E. (1971). International corporations: The industrial economics of foreign investment. Economica, 38, pp. 1-27.

Chandler, A. (1990). Scale and scope. The dynamics of industrial capitalism. Cambridge, Mass., Harvard/Belknap.

Claver, E.; Molina, J. \& Quer, D. (2000). Un enfoque estratégico para la internacionalización de la empresa familiar. I Congreso Nacional de Investigación sobre la Empresa Familiar, Instituto de Investigación de la Empresa Familiar. Valencia, septiembre, pp. 419-447.

Claver, E.; Rienda, L. \& Quer, D. (2007). Incide el carácter familiar en el compromiso internacional de las empresas españolas, Investigaciones Europeas de Dirección y Economía de la Empresa, 13, (3), pp. 13-32.

Collis, D.J. (1991). A resource-based analysis of global competition: The case of the bearings industry. Strategic Management Journal, 12 (especial, verano), pp. 49-68.

Daily, C.M. \& Dollinger, M.J. (1992). An empirical examination of ownership structure in family and professionally managed firms. Family Business Review, 5,(2), pp. $117-136$.

Davis, P. \& Harveston, P. (2000). Internationalization and organizational growth: The impact of internet usage and technology involvement among entrepreneurled family business. Family Business Review, 13(2), pp. 107-120.

Donckels, R. \& Lambrecht, J. (1999). Are family businesses really different? What we know from Western European business research that could be applied to the reemergence of family-based. Enterprises in East Central Europe. Family Business Review, 12(2), pp. 171-191.

Dunning, J.H. (1973). The determinants of international production. Oxford Economic Papers, 25, pp. 289-336. 
Durán, J.J. (2005). Teoría de la economía y dirección de la empresa multinacional, en Durán (coord.): La empresa multinacional española. Estrategias y ventajas competitivas. Minerva Ediciones, Madrid, pp. 11-46.

Fernandez-Ortiz, R. (2005). Análisis sistémico del compromiso exportador de la pyme familiar: perfil de recursos y capacidades de la pyme exportador riojana. Departamento de Economía y Empresas. Universidad de La Rioja.

Fernández, Z. \& Nieto, M.J. (2005). Internationalization strategy of small and medium-sized family businesses: Some influential factors. Family Business Review, 18(1), pp. 77-89.

Fuentes Lombardo, G. (2006). La influencia del carácter familiar de la empresa en su estrategia de internacionalización: Una aplicación al sector vitivinícola con denominación de origen. Departamento de Administración de Empresas, Contabilidad y Sociología. Universidad de Jaén.

Gallo, M. \& García-Pont, C. (1996). Important factors in family business internationalization. Family Business Review, 9(1), pp. 45-60.

Gallo, M. Y Luostarinem R. (1993). Internacionalización: Un cambio desafiante para las empresas familiares. En GaIlo, M.: La empresa familiar 4. Estudio y ediciones IESE, S.L., 91-101.

Gallo, M. \& Sveen, J. (1991). Internationalizing the family business: Facilitating and retraining factors, Family Business Review, 4(2), pp. 181-190.

Gudmundson, D.; Tower, C. \& Hartman, A. (2003). Innovation in small businesses: Culture and ownership structure do matter, Journal of Developmental Entrepreneurship, 18(1), pp. 1-17.

Guillén, M. (2001). International business. En International Encyclopedia of the Social and Behavioral Sciences. Oxford: Elsevier Publishers, pp. 7768-7771.

Hall, R. (1992). The strategic analysis of intangible resources. Strategic Management Journal, 13(2), pp.135-144.

Harris, R., Martínez, J. \&Ward, J. (1994). Is strategy different for the family-owned business? Family Business Review, 7(2), pp. 159-176.

Horton, T.P. (1986). Managing in a family way. Management Review, 75(2), p. 3.
Hufbauer, O. (1975). The multinational corporation and direct investment, en Kenen, P.B. (eds.): International Trade and Finance, Cambridge University Press, Cambridge.

Hymer, S. (1976). The international operations of national firms: A study of direct foreign investment. MIT Press.

Ibrahim, A. \& Ellis, W. (1994). Family business management. Concepts and practice. Kendall/Hunt Publishing Company, Dubuque.

Ibrahim, A.; Soufani, K. \& Lam, J. (2001). A study of succession in a family firm. Family Business Review, 14(3), pp. 245-258.

James, H. (1999). What can the family contribute to business? Examining contractual relationships. Family Business Review, 12(1), pp. 61-72.

Johanson, J. \& Vahlne, J.E. (1977). The internationalisation process of the firms-a model of knowledge development and increasing foreign market commitment. Journal of International Business Studies, 8(1), pp. 23-32.

Jones, M.V. \& Coviello, N.E. (2005). Internationasition: conceptualising an entrepreneurial process of behaviour in time, Journal of International Business Studies, 36, pp. 284-303

Kets De Vries, M. (1993). Lo bueno y lo malo de las empresas de titularidad familiar, Harvard Deusto Business Review, 4, pp. 32-44.

Kets De Vries, M. (1996). Family Business: Human Dilemmas in the Family Firm. Thomson Business Press, London.

Knickerbocker, F.T. (1973). Oligopolistic reaction and the multinational enterprise. MA: Harvard University Press.

Knight, G.A. \& Cavusgil, S.T. (2004). Innovation, organizational capabilities, and the born-global firm, Journal of International Business Studies, 35, pp. 124-141

Kogut, B. (1983). Foreign direct investment as a sequential process, en: Kindleberger C.P., Audretsch D. (eds.) The Multinational Corporation in the 1980s. MIT Press, Cambridge, MA.

Kogut, B. \& Kulatilaka, N. (1993). Operating flexibility, global manufacturing, and the option value of a multinational network. Management Science, 39(11), pp. 123-139.

Lansberg, I. (1983). Managing human resources in family firms: The problem of institutional overlap, Organizational Dynamics, 12(1), pp. 39-46. 
Leach, P.\& Bogod, T. (1999). The BDO-stoy hayward guide to the family business. Kogan Page, London.

Mcconaughy, D:; Matthews, C. \& Fialko, A. (2001). Founding family controlled firms: Performance, risk, and value, Journal of Small Business Management, 39(1), pp. 31-49.

Mcwhinney, W. (1988). Entrepreneurs, owners, and stewards: The conduct of a family business, New Management, 6(1), pp. 4-11.

Morck, R. \& Yeung, B. (2003). Agency problems in large family business groups, Entrepreneurship: Theory and Practice, 27(4), pp. 367-382.

Nieto, M. (1999). Decisiones estratégicas de la empresa familiar: Innovación e internacionalización. IX Congreso Nacional ACEDE. Burgos. 12, 13 y 14 de Septiembre.

Okoroafo, S. (1999). Internationalization of family business: Evidence from Northwest Ohio, U.S.A.. Family Business Review, 12(2), pp. 147-158.

Poza, E. (1995). A la sombra del roble: La empresa privada familiary su continuidad. Editorial Universitaria para la Empresa Familiar, Ohio.

Poza, E.; Alfred, T. \& Maheshwari, A. (1997). Stakeholder perceptions of culture and management practices in family and family firms: A preliminary report. Family Business Review, 10(2), pp. 135-155.

Rialp, A., Rialp, J. \& Knight, G.A. (2005). The phenomenon of early internationalising firms: what do we know after a decade (1993-2003) of scientific inquiry?, International Business Review, 14, pp. 147-166.

Rialp, A. \& Rialp, J. (2005). El fenómeno de las empresas rápidamente internacionalizadas. Una revisión comentada de la literatura a nivel internacional y nacional. En Durán Herrera, J.J. (ed.) La multinacionalización de la empresa española. Estrategias y ventajas competitivas. Madrid: Minerva ediciones, pp. 47-82.

Romano, C.; Tanewski, G. \& Smyrnios, K. (2000). Capital structure decision making: A model for family business. Journal of Business Venturing, 16(3), pp. 285-311.
Sorenson, R. (2000). The contribution of leadership style and practices to family and business success. Family Business Review, 13(3), pp.183-200.

Steier, L. (2001). Family firms, plural forms of governance, and the evolving role of trust. Family Business Review, 14(4), pp. 353-367.

Tagiuri, R. \& Davis, J. A. (1996). Bivalent attributtes of the family firm. Family Business Review, 9 (2), pp.199-208.

Tallman, S. (1991). Strategic management model and resource-based strategies among MNEs in a host market, Strategic Management Journal, 12, pp. 69-82.

Taylor, S.J. \& Bogdan, R. (1992). Introducción a los métodos cualitativos de investigación: La búsqueda de significados. Paidos, Barcelona.

Vallejo, M. (2003). La cultura de la empresa familiar como ventaja competitiva: Una aplicación al sector de concesionarios de automoción. Tesis doctoral no publicada. Departamento de Administración de Empresas, Contabilidad y Sociología, Universidad de Jaén.

Vernon, R. (1966). International investment and international trade in the product cycle. Quarterly Journal of Economics, 80(1), pgs. 190-207.

Ward, J.L. (1997). Growing the family business: Special challenges and best practices. Family Business Review, 10(4), pp. 323-337.

Westhead, P. (1997). Ambitions, 'external' environment and strategic factor differences between family and non-family companies. Entrepreneurship \& Regional Development, 9(2), pp. 127-157.

Yeung, H. (2000). Limits to the growth of family-owned business? The case of Chinese transnational corporations from Hong Kong. Family Business Review, 13(1), pp. 55-70.

Zahra, S.A. (2003). International expansion of U.S. manufacturing family businesses: The effect of ownership and involvement. Journal of Business Venturing, 18(4), pp. 495-512.

RECIBIDO: 16-09-2009

ACEPTADO: 19-11-2009 